\title{
White South American
}

National Cancer Institute

\section{Source}

National Cancer Institute. White South American. NCI Thesaurus. Code C128994.

A person having origins in the any of the countries of South America who identifies as white. 